\title{
Ramsey's Theorem
}

\author{
Marco Riccardi \\ Casella Postale 49 \\ 54038 Montignoso, Italy
}

\begin{abstract}
Summary. The goal of this article is to formalize two versions of Ramsey's theorem. The theorems are not phrased in the usually pictorial representation of a coloured graph but use a set-theoretic terminology. After some useful lemma, the second section presents a generalization of Ramsey's theorem on infinite set closely following the book [9]. The last section includes the formalization of the theorem in a more known version (see [1]).
\end{abstract}

MML identifier: RAMSEY_1, version: $\underline{7.9 .01 \quad 4.101 .1015}$

The notation and terminology used here are introduced in the following papers: [15], [16], [17], [4], [3], [6], [12], [7], [2], [5], [8], [14], [13], [10], and [11].

\section{Preliminaries}

For simplicity, we adopt the following convention: $n, m, k$ are natural numbers, $X, Y, Z$ are sets, $f$ is a function from $X$ into $Y$, and $H$ is a subset of $X$.

Let us consider $X, Y, H$ and let $P$ be a partition of $[X]^{Y}$. We say that $H$ is homogeneous for $P$ if and only if:

(Def. 1) There exists an element $p$ of $P$ such that $[H]^{Y} \subseteq p$.

Let us consider $n$ and let $X$ be an infinite set. One can check that $[X]^{n}$ is non empty.

Let us consider $n, X, Y, f$. Let us assume that $f$ is one-to-one and $\overline{\bar{n}} \subseteq \overline{\bar{X}}$ and $X$ is non empty and $Y$ is non empty. The functor $f \|^{n}$ yields a function from $[X]^{n}$ into $[Y]^{n}$ and is defined by:

(Def. 2) For every element $x$ of $[X]^{n}$ holds $\left(f \|^{n}\right)(x)=f^{\circ} x$.

Next we state four propositions: 
(1) If $f$ is one-to-one and $\overline{\bar{n}} \subseteq \overline{\bar{X}}$ and $X$ is non empty and $Y$ is non empty, then $\left[f^{\circ} H\right]^{n}=\left(f \|^{n}\right)^{\circ}\left([H]^{n}\right)$.

(2) If $X$ is infinite and $X \subseteq \omega$, then $\overline{\bar{X}}=\omega$.

(3) If $X$ is infinite, then $X \cup Y$ is infinite.

(4) If $X$ is infinite and $Y$ is finite, then $X \backslash Y$ is infinite.

Let $X$ be an infinite set and let $Y$ be a set. Note that $X \cup Y$ is infinite.

Let $X$ be an infinite set and let $Y$ be a finite set. One can verify that $X \backslash Y$ is infinite.

The following propositions are true:

(5) $[X]^{0}=\{0\}$.

(6) For every finite set $X$ such that $\operatorname{card} X<n$ holds $[X]^{n}$ is empty.

(7) If $X \subseteq Y$, then $[X]^{Z} \subseteq[Y]^{Z}$.

(8) If $X$ is finite and $Y$ is finite and $\overline{\bar{Y}}=X$, then $[Y]^{X}=\{Y\}$.

(9) If $X$ is non empty and $Y$ is non empty, then $f$ is constant iff there exists an element $y$ of $Y$ such that $\operatorname{rng} f=\{y\}$.

(10) For every finite set $X$ such that $k \leq \operatorname{card} X$ there exists a subset $Y$ of $X$ such that $\operatorname{card} Y=k$.

(11) If $m \geq 1$, then $n+1 \leq\left(\begin{array}{c}n+m \\ m\end{array}\right)$.

(12) If $m \geq 1$ and $n \geq 1$, then $m+1 \leq\left(\begin{array}{c}n+m \\ m\end{array}\right)$.

(13) Let $X$ be a non empty set, $p_{1}, p_{2}$ be elements of $X, P$ be a partition of $X$, and $A$ be an element of $P$. Suppose $p_{1} \in A$ and (the projection onto $P)\left(p_{1}\right)=($ the projection onto $P)\left(p_{2}\right)$. Then $p_{2} \in A$.

\section{Infinite Ramsey Theorem}

We now state two propositions:

(14) Let $F$ be a function from $[X]^{n}$ into $k$. Suppose $k \neq 0$ and $X$ is infinite. Then there exists $H$ such that $H$ is infinite and $F\left\lceil[H]^{n}\right.$ is constant.

(15) Let $X$ be an infinite set and $P$ be a partition of $[X]^{n}$. If $\overline{\bar{P}}=k$, then there exists a subset of $X$ which is infinite and homogeneous for $P$.

\section{RAMSEY'S THEOREM}

The scheme BinInd2 concerns a binary predicate $\mathcal{P}$, and states that:

$$
\mathcal{P}[m, n]
$$

provided the following conditions are satisfied:

- $\mathcal{P}[0, n]$ and $\mathcal{P}[n, 0]$, and

- If $\mathcal{P}[m+1, n]$ and $\mathcal{P}[m, n+1]$, then $\mathcal{P}[m+1, n+1]$.

We now state two propositions: 
(16) Suppose $m \geq 2$ and $n \geq 2$. Then there exists a natural number $r$ such that

(i) $\quad r \leq\left(\begin{array}{c}(m+n)-^{\prime} 2 \\ m-^{\prime} 1\end{array}\right)$,

(ii) $r \geq 2$, and

(iii) for every finite set $X$ and for every function $F$ from $[X]^{2}$ into Seg 2 such that card $X \geq r$ there exists a subset $S$ of $X$ such that card $S \geq m$ and $\operatorname{rng}\left(F \uparrow[S]^{2}\right)=\{1\}$ or card $S \geq n$ and $\operatorname{rng}\left(F \uparrow[S]^{2}\right)=\{2\}$.

(17) Let $m$ be a natural number. Then there exists a natural number $r$ such that for every finite set $X$ and for every partition $P$ of $[X]^{2}$ if card $X \geq r$ and $\overline{\bar{P}}=2$, then there exists a subset $S$ of $X$ such that card $S \geq m$ and $S$ is homogeneous for $P$.

\section{REFERENCES}

[1] M. Aigner and G. M. Ziegler. Proofs from THE BOOK. Springer-Verlag, Berlin Heidelberg New York, 2004.

[2] Grzegorz Bancerek. Cardinal numbers. Formalized Mathematics, 1(2):377-382, 1990.

[3] Grzegorz Bancerek and Krzysztof Hryniewiecki. Segments of natural numbers and finite sequences. Formalized Mathematics, 1(1):107-114, 1990.

[4] Czesław Byliński. Functions and their basic properties. Formalized Mathematics, 1(1):5565, 1990.

[5] Czesław Byliński. Functions from a set to a set. Formalized Mathematics, 1(1):153-164, 1990.

[6] Czesław Byliński. Partial functions. Formalized Mathematics, 1(2):357-367, 1990.

[7] Agata Darmochwał. Finite sets. Formalized Mathematics, 1(1):165-167, 1990.

[8] Krzysztof Hryniewiecki. Basic properties of real numbers. Formalized Mathematics, 1(1):35-40, 1990.

[9] T. J. Jech. Set Theory. Springer-Verlag, Berlin Heidelberg New York, 2002.

[10] Rafał Kwiatek. Factorial and Newton coefficients. Formalized Mathematics, 1(5):887-890, 1990.

[11] Takaya Nishiyama and Yasuho Mizuhara. Binary arithmetics. Formalized Mathematics, 4(1):83-86, 1993.

[12] Konrad Raczkowski and Paweł Sadowski. Equivalence relations and classes of abstraction. Formalized Mathematics, 1(3):441-444, 1990.

[13] Marco Riccardi. The sylow theorems. Formalized Mathematics, 15(3):159-165, 2007.

[14] Andrzej Trybulec. A Borsuk theorem on homotopy types. Formalized Mathematics, 2(4):535-545, 1991.

[15] Zinaida Trybulec. Properties of subsets. Formalized Mathematics, 1(1):67-71, 1990.

[16] Edmund Woronowicz. Relations and their basic properties. Formalized Mathematics, 1(1):73-83, 1990.

[17] Edmund Woronowicz. Relations defined on sets. Formalized Mathematics, 1(1):181-186, 1990. 